\title{
Genetic response to metabolic fluctuations: correlation between central carbon metabolism and DNA replication in Escherichia coli
}

Monika Maciąg ${ }^{1}$, Dariusz Nowicki', Laurent Janniere², Agnieszka Szalewska-Pałasz ${ }^{1}$ and Grzegorz Węgrzyn ${ }^{1 *}$

\begin{abstract}
Background: Until now, the direct link between central carbon metabolism and DNA replication has been demonstrated only in Bacillus. subtilis. Therefore, we asked if this is a specific phenomenon, characteristic for this bacterium and perhaps for its close relatives, or a more general biological rule.

Results: We found that temperature-sensitivity of mutants in particular genes coding for replication proteins could be suppressed by deletions of certain genes coding for enzymes of the central carbon metabolism. Namely, the effects of dnaA46(ts) mutation could be suppressed by dysfunction of pta or ackA, effects of dnaB(ts) by dysfunction of pgi or pta, effects of $d n a E 486(\mathrm{ts})$ by dysfunction of $t k t B$, effects of $d n a G(\mathrm{ts})$ by dysfunction of gpmA, pta or ackA, and effects of dnaN159(ts) by dysfunction of pta or ackA. The observed suppression effects were not caused by a decrease in bacterial growth rate.
\end{abstract}

Conclusions: The genetic correlation exists between central carbon metabolism and DNA replication in the model Gram-negative bacterium, E. coli. This link exists at the steps of initiation and elongation of DNA replication, indicating the important global correlation between metabolic status of the cell and the events leading to cell reproduction.

\section{Background}

When considering a bacterial cell as a microbial factory, producing various macromolecules either natural or formed due to introduction of recombinant genes, several biochemical processes must be taken into consideration. Among them, there are two basic processes ensuring that more specialized reactions (like transcription of particular genes and translation of particular mRNAs on ribosomes as well as enzyme-mediated production of various compounds) can occur. These two processes are central carbon metabolism (for a review see ref. [1]) and DNA replication (for a review see ref. [2]). The former one provides energy from nutrients, which is absolutely necessary to all life functions of cells. The latter one, although consuming cellular energy, ensures integrity of genetic material and its inheritance by daughter cells after each cell division,

\footnotetext{
* Correspondence: wegrzyn@biotech.univ.gda.pl

'Department of Molecular Biology, University of Gdańsk, Kładki 24, 80-822 Gdańsk, Poland

Full list of author information is available at the end of the article
}

providing the source of information about biological structures and functions of macromolecules.

The central carbon metabolism (CCM) is generally recognized as a set of biochemical pathways devoted to transport and oxidation of main carbon sources in the cell [1]. In a model Gram-negative bacterium, Escherichia coli, it consists of the phosphortransferase system, glycolysis, gluconeogenesis, pentose-monophosphate bypass with Entner-Dudoroff pathway, Krebs cycle with glyoxylate bypass and the respiration chain [3]. Biochemical reactions of these pathways ensure the optimal energy production and usage in the cell at particular growth conditions, in order to keep homeostasis.

DNA replication is a process of genetic information duplication, which is necessary to equal and precise distribution of the genetic material to both daughter cells after each cell division [2]. The process of replicative DNA synthesis requires large cellular machinery, which in E. coli consists of DNA polymerase III holoenzyme (containing at least 10 subunits) and other essential proteins, including DnaB helicase and DnaG primase.
C Biomed Central

() 2011 Maciąag et al; licensee BioMed Central Ltd. This is an Open Access article distributed under the terms of the Creative Commons Attribution License (http://creativecommons.org/licenses/by/2.0), which permits unrestricted use, distribution, and reproduction in any medium, provided the original work is properly cited. 
Additional proteins (DnaA, DnaC) are required for DNA replication initiation at a specific genome region, called oriC $[2,4]$.

Although it was observed previously that regulation of DNA replication may depend on bacterial cell metabolism, it was generally assumed that this dependency is indirect. For example, it might result from different availability of cellular energy and/or precursors of macromolecules $[5,6]$ or from production of specific alarmons, like cyclic AMP (cAMP) $[7,8]$ or guanosine tetraphosphate (ppGpp) [9-12], in response to nutritional deprivations. However, it was reported recently that DNA replication may be directly linked to central carbon metabolism, particularly glycolysis, in a model Gram-positive bacterium, Bacillus subtilis [13]. Namely, specific suppression of conditionally-lethal (temperaturesensitive, ts) mutations in genes coding for replication proteins (DnaE, a DNA polymerase involved in lagging strand synthesis, DnaC, a helicase - homologue of E. coli DnaB protein, and DnaG, the primase) by dysfunction of certain genes coding for enzymes involved in glycolysis, was observed. An indirect suppression mechanism (e.g. by slowing down of bacterial growth rate) was excluded, strongly suggesting a real link between glycolysis and DNA replication. Thus, the existence of such a link should be considered in any studies on both these processes, as well as when constructing and using biotechnological systems for efficient production of desired compounds.

Until now, the direct link between central carbon metabolism and DNA replication has been demonstrated only in B. subtilis [13]. Therefore, we asked if this is a specific phenomenon, characteristic for this bacterium and perhaps for its close relatives, or a more general biological rule. Since E. coli is both a model Gram-negative bacterium and a widely used host for production of recombinant proteins, in our studies, which were performed to answer the above question, we employed strains of this species.

\section{Methods}

\section{Bacterial strains, plasmids and bacteriophages}

E. coli strains used in this work are listed in Table 1. Plasmids and bacteriophages are described in Table 2. New bacterial strains and plasmids were constructed according to standard procedures of P1 transduction and molecular cloning, respectively [14].

\section{Oligonucleotides}

Oligunucleotides are described in Table 3.

\section{Growth conditions}

Luria -Bertani (LB) medium, and minimal media M9 and MM, were used [14]. Solid media contained $1.5 \%$ of bacteriological agar. For liquid cultures, bacteria were grown in various media in shake flasks, with aeration (by shaking). Overnight cultures were diluted in LB and grown to $\mathrm{OD}_{600}=0.3$. Then, $100 \mu \mathrm{l}$ of the culture or its dilution was plated on solid media. The plates were then incubated at indicated temperatures for indicated time. CFU (colony forming units) were calculated from plates where colony number was between 100 and 1000 .

\section{Results}

We have employed six E. coli temperature-sensitive mutants in following genes coding for proteins necessary for chromosomal DNA replication: dnaA (coding for the replication initiator protein that binds to the oriC region and forms a specific nucleoprotein structure; this is the first step in the DNA replication initiation), dnaB (coding for the main DNA helicase, the enzyme necessary to melt DNA during the replication process), dnaC (coding for the protein which delivers DnaB helicase to the DnaA protein bound to oriC), dnaE (coding for the $\alpha$ subunit of DNA polymerase III, the catalytic subunit of this enzyme), dnaG (coding for primase, an enzyme necessary to synthesize RNA primers during DNA replication) and $\operatorname{dnaN}$ (coding for the $\beta$ subunit of DNA polymerase III, a protein forming the sliding clamp and allowing DNA polymerase III to be kept on the template DNA strand when synthesizing new polynucleotide strand) [for more detailed information on these genes and their products, see ref. 2]. These mutants are described in Table 1.

To test whether mutations (particularly deletioninsertion mutations) in genes coding for enzymes from central carbon metabolism (CCM) may suppress temperature sensitivity of the replication mutants, we have determined the sensitivity profiles of all tested conditionally lethal mutants. This was necessary to chose temperatures that severely restricted growth of mutant cells, however, which still allowed observing some viability of tested strains; otherwise detection of any suppression would be impossible, as observed in the B. subtilis study [13]. The profiles of temperature-sensitivity of dnaA, dnaB, dnaC, dnaE, dnaG and dnaN mutants in LB medium are shown in Figure 1.

A series of double mutants, bearing mutations in one of the replication genes and in one of genes coding for CCM enzyme, has been constructed by P1 transduction (Table 1). For these constructions, deletion-insertion mutants in following genes were employed: gapC, pykF, tpiA, pgi, fbaB, gpmA, pck, zwf, tktB, pta, ackA, aceB, $a c n B$, and $i c d$. Enzymes encoded by these genes are listed in Table 4, and locations (in particular biochemical pathways) of reactions catalyzed by them are marked on the scheme depicting the central carbon metabolism in E. coli (Figure 2). 
Table $1 E$. coli strains used in this work

\begin{tabular}{|c|c|c|}
\hline Strain & Relevant characteristics & Reference or source \\
\hline JJC809 (PC8) & 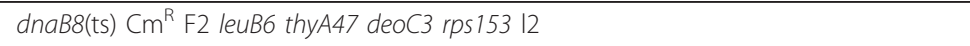 & [21] \\
\hline PC2 & dnaC(ts) thy leu rpsL & [21] \\
\hline PC3 & dnaG(ts) Hfr leu thy rpsL & [22] \\
\hline MG1655 & $F-\lambda-i l v G-r f b-50 r p h-1$ & [23] \\
\hline MG1655dnaA46 & F- $\lambda$ - ilvG-rfb-50 rph-1 dnaA46 tna::Tn10 & [24] \\
\hline $\mathrm{DH} 5 \alpha$ & $\begin{array}{l}\text { F- } \phi 80 \text { lacZIM15 } \triangle \text { (lacZYA-argF)U169 deoR recA1 endA1 hsdR17(rk-, mk+) phoA supE44 } \\
\text { thi-1 gyrA96 relA1 } \lambda^{-}\end{array}$ & [25] \\
\hline BW25113 & $\Delta(\operatorname{araD}-\operatorname{araB}) 567, \Delta$ lacZ4787::rrnB-3, $\lambda$; rph-1, $\Delta(r h a D-r h a B) 568$, hsdR514 & [26] \\
\hline JW1122 & Same as BW25113 but $\Delta i c d:: k a n$ & [27] \\
\hline JW1413 & Same as BW25113 but $\triangle g a p C:: k a n$ & [27] \\
\hline JW1666 & Same as BW25113 but ApykF::kan & {$[27]$} \\
\hline JW1841 & Same as BW25113 but $\Delta$ wf::kan & {$[27]$} \\
\hline JW2449 & Same as BW25113 but $\Delta t k t B: . k a n$ & [27] \\
\hline JW3366 & Same as BW25113 but $\triangle p c k:: k a n$ & {$[27]$} \\
\hline JW3890 & Same as BW25113 but $\triangle$ tpiA.:kan & [27] \\
\hline JW3974 & Same as BW25113 but $\triangle a c e B:: k a n$ & {$[27]$} \\
\hline JW3985 & Same as BW25113 but Apgi::kan & {$[27]$} \\
\hline JW2294 & Same as BW25113 but $\triangle p t a:$ :kan & [27] \\
\hline JW2293 & Same as BW25113 but $\triangle a c k A .: k a n$ & {$[27]$} \\
\hline JW5173 & Same as BW25113 but $\Delta i c d C:: k a n$ & [27] \\
\hline JW5344 & Same as BW25113 but $\triangle f b a B:: k a n$ & [27] \\
\hline JW0738 & Same as BW25113 but $\Delta g p m A:: k a n$ & [27] \\
\hline NR13339 & Same as KA796 with dnaN159(Ts) zid501::Tn10 & [28] \\
\hline NR7651 & Same as MC4100 lacZ104 dnaE486(Ts) zae502::Tn10 & {$[28]$} \\
\hline
\end{tabular}

AS701 MG1655 dnaA46 $\triangle a c n:: k a n$

AS702 MG1655 dnaA46 $\Delta i c d: k$ kan

AS703 MG1655 dnaA46 $\triangle$ gapC::kan

AS704 MG1655 dnaA46 $\Delta p y k F: . k a n$

AS705 MG1655 dnaA46 $\Delta z w f: . K a n$

AS706 MG1655 dnaA46 AtktB::kan

AS707 MG1655 dnaA46 Apck::Kan

AS708 MG1655 dnaA46 $\triangle$ tpiA::Kan

AS709 MG1655 dnaA46 $\triangle a c e B: K a n$

AS710 MG1655 dna A46 Apgi:.kan

AS711 MG1655 dna A46 spta::kan

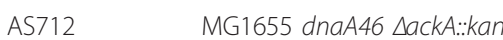

AS713 MG1655 dnaA46 $\Delta i c d C:: k a n$

AS714 MG1655 dnaA46 AfbaB::kan

AS715 MG1655 dnaA46 IgpmA::kan

AS766 MG1655 dnaB8 $\triangle a c n: k a n$

AS767 MG1655 dnaB8 $\Delta i c d:: k a n$

AS768 MG1655 dnaB8 $\Delta$ gapC::kan

AS769 MG1655 dnaB8 $\triangle$ pykF::kan

AS770 MG1655 dnaB8 $\Delta z w f: . k a n$

AS771 MG1655 dnaB8 $\triangle$ tktB::kan

AS772 MG1655 dnaB8 Apck:kan

AS773 MG1655 dnaB8 $\Delta$ tpiA::kan

AS774 MG1655 dnaB8 $\triangle a c e B:: k a n$

AS775 MG1655 dnaB8 spgi::kan

AS776 MG1655 dnaB8 $\Delta$ tata:kan

AS778 MG1655 dnaB8 $\triangle a c k A: . k a n$

This study, by P1 transduction from JW0114 This study, by P1 transduction from JW1122 This study, by P1 transduction from JW1413 This study, by P1 transduction from JW1666 This study, by P1 transduction from JW1841 This study, by P1 transduction from JW2449 This study, by P1 transduction from JW3366 This study, by P1 transduction from JW3890 This study, by P1 transduction from JW3974 This study, by P1 transduction from JW3985 This study, by P1 transduction from JW2294 This study, by P1 transduction from JW2293 This study, by P1 transduction from JW5173 This study, by $\mathrm{P} 1$ transduction from JW5344 This study, by P1 transdukcion from JW0738

This study, by P1 transduction from JW0114 This study, by P1 transduction from JW1122 This study, by P1 transduction from JW1413 This study, by P1 transduction from JW1666 This study, by P1 transduction from JW1841 This study, by P1 transduction from JW1841 This study, by P1 transduction from JW3366 This study, by P1 transduction from JW3890 This study, by P1 transduction from JW3974 This study, by P1 transduction from JW3985 This study, by P1 transduction from JW2294 This study, by P1 transduction from JW2293 
Table 1 E. coli strains used in this work (Continued)

\begin{tabular}{|c|c|c|}
\hline AS779 & MG1655 dnaB8 $\Delta i c d C:: k a n$ & This study, by P1 transduction from JW5173 \\
\hline AS780 & MG1655 dnaB8 $\triangle f b a B:: k a n$ & This study, by P1 transduction from JW5344 \\
\hline AS781 & MG1655 dnaB8 $\Delta g p m A:: k a n$ & This study, by P1 transduction from JW0738 \\
\hline AS750 & PC2 dnaC(ts) $\triangle a c n:: k a n$ & This study, by P1 transduction from JW0114 \\
\hline AS751 & PC2 dnaC(ts) $\Delta i c d:: k a n$ & This study, by P1 transduction from JW1122 \\
\hline AS752 & PC2 dnaC(ts) $\Delta g a p C: .: k a n$ & This study, by P1 transduction from JW1413 \\
\hline AS753 & PC2 dnaC(ts) $\Delta p y k F .: k a n$ & This study, by P1 transduction from JW1666 \\
\hline AS754 & PC2 dnaC(ts) $\Delta w f:: k a n$ & This study, by P1 transduction from JW1841 \\
\hline AS755 & PC2 dnaC(ts) $\Delta t k t B:: k a n$ & This study, by P1 transduction from JW2449 \\
\hline AS756 & PC2 dnaC(ts) spck::kan & This study, by P1 transduction from JW3366 \\
\hline AS757 & PC2 dnaC(ts) $\Delta t p i A:: k a n$ & This study, by P1 transduction from JW3890 \\
\hline AS758 & PC2 dnaC(ts) $\triangle a c e B:: k a n$ & This study, by P1 transduction from JW3974 \\
\hline AS759 & PC2 dnaC(ts) spgi::kan & This study, by P1 transduction from JW3985 \\
\hline AS760 & PC2 dnaC(ts) spta::kan & This study, by P1 transduction from JW2294 \\
\hline AS761 & PC2 dnaC(ts) $\triangle a c k A:: k a n$ & This study, by P1 transduction from JW2293 \\
\hline AS762 & PC2 dnaC(ts) $\Delta i c d C:: k a n$ & This study, by P1 transduction from JW5173 \\
\hline AS763 & PC2 dnaC(ts) $\Delta f b a B: . k a n$ & This study, by P1 transduction from JW5344 \\
\hline AS764 & PC2 dnaC(ts) $\Delta g p m A .: k a n$ & This study, by P1 transduction from JW0738 \\
\hline AS783 & PC3 dnaG(ts) $\Delta a c n:: k a n$ & This study, by P1 transduction from JW0114 \\
\hline AS784 & PC3 dnaG(ts) $\Delta i c d:: k a n$ & This study, by P1 transduction from JW1122 \\
\hline AS785 & PC3 dnaG(ts) $\Delta g a p C:: k a n$ & This study, by P1 transduction from JW1413 \\
\hline AS786 & PC3 dnaG(ts) $4 p y k F:: k a n$ & This study, by P1 transduction from JW1666 \\
\hline AS787 & PC3 dnaG(ts) $\Delta w f:: k a n$ & This study, by P1 transduction from JW1841 \\
\hline AS788 & PC3 dnaG(ts) $\Delta t k t B:: k a n$ & This study, by P1 transduction from JW2449 \\
\hline AS789 & PC3 dnaG(ts) 4pck::kan & This study, by P1 transduction from JW3366 \\
\hline AS790 & PC3 dnaG(ts) $\Delta t p i A:: k a n$ & This study, by P1 transduction from JW3890 \\
\hline AS791 & PC3 dnaG(ts) $\triangle a c e B:: k a n$ & This study, by P1 transduction from JW3974 \\
\hline AS792 & PC3 dnaG(ts) spgi::kan & This study, by P1 transduction from JW3985 \\
\hline AS793 & PC3 dnaG(ts) spta::kan & This study, by P1 transduction from JW2294 \\
\hline AS794 & PC3 dnaG(ts) $\triangle a c k A:: k a n$ & This study, by P1 transduction from JW2293 \\
\hline AS795 & PC3 dnaG(ts) $\Delta i c d C: . k a n$ & This study, by P1 transduction from JW7173 \\
\hline AS796 & PC3 dnaG(ts) $\Delta f b a B:: k a n$ & This study, by P1 transduction from JW5344 \\
\hline AS797 & PC3 dnaG(ts) $\Delta g p m A:: k a n$ & This study, by P1 transduction from JW0738 \\
\hline AS718 & MG1655 dnaE486 $\triangle a c n$ & This study, by P1 transduction from JW0114 \\
\hline AS719 & MG1655 dnaE486 $\Delta i c d$ & This study, by P1 transduction from JW1122 \\
\hline AS720 & MG1655 dnaE486 $\triangle g a p C$ & This study, by P1 transduction from JW1413 \\
\hline AS721 & MG1655 dnaE486 ApykF & This study, by P1 transduction from JW1666 \\
\hline AS722 & MG1655 dnaE486 $\Delta z w f$ & This study, by P1 transduction from JW1841 \\
\hline AS723 & MG1655 dnaE486 $\triangle t k t B$ & This study, by P1 transduction from JW2449 \\
\hline AS724 & MG1655 dnaE486 $\Delta p c k$ & This study, by P1 transduction from JW3366 \\
\hline AS725 & MG1655 dnaE486 $\triangle t p i A$ & This study, by P1 transduction from JW3890 \\
\hline AS726 & MG1655 dnaE486 $\triangle a c e B$ & This study, by P1 transduction from JW3974 \\
\hline AS728 & MG1655 dnaE486 Apgi & This study, by P1 transduction from JW3985 \\
\hline AS729 & MG1655 dnaE486 $4 p t a$ & This study, by P1 transduction from JW2294 \\
\hline AS730 & MG1655 dnaE486 $\triangle a c k A$ & This study, by P1 transduction from JW2293 \\
\hline AS731 & MG1655 dnaE486 $\triangle i c d C$ & This study, by P1 transduction from JW5173 \\
\hline AS732 & MG1655 dnaE486 $\triangle f b a B$ & This study, by P1 transduction from JW5344 \\
\hline AS733 & MG1655 dnaE486 $\triangle g p m A$ & This study, by P1 transduction from JW0738 \\
\hline AS734 & MG1655 dnaN159 $\triangle a c n B:: k a n$ & This study, by P1 transduction from JW0114 \\
\hline AS735 & MG1655 dnaN159 $\Delta i c d:: k a n$ & This study, by P1 transduction from JW1122 \\
\hline AS736 & MG1655 dnaN159 $\Delta g a p C:: k a n$ & This study, by P1 transduction from JW1413 \\
\hline AS737 & MG1655 dnaN159 spykF::kan & This study, by P1 transduction from JW1666 \\
\hline
\end{tabular}


Table 1 E. coli strains used in this work (Continued)

\begin{tabular}{|c|c|c|}
\hline AS738 & MG1655 dnaN159 মzwf::kan & This study, by P1 transduction from JW1841 \\
\hline AS739 & MG1655 dnaN159 $\Delta t k t B: * k a n$ & This study, by P1 transduction from JW2449 \\
\hline AS740 & MG1655 dnaN159 spck::kan & This study, by P1 transduction from JW3366 \\
\hline AS741 & MG1655 dnaN159 $\Delta t p i A .: k a n$ & This study, by P1 transduction from JW3890 \\
\hline AS742 & MG1655 dnaN159 $\Delta a c e B:: k a n$ & This study, by P1 transduction from JW3974 \\
\hline AS743 & MG1655 dnaN159 spgi::kan & This study, by P1 transduction from JW3985 \\
\hline AS744 & MG1655 dnaN159 4pta::kan & This study, by P1 transduction from JW2294 \\
\hline AS745 & MG1655 dnaN159 sackA::kan & This study, by P1 transduction from JW2293 \\
\hline AS746 & MG1655 dnaN159 $\Delta i c d C:: k a n$ & This study, by P1 transduction from JW5173 \\
\hline AS747 & MG1655 dnaN159 $\Delta$ fbaB::kan & This study, by P1 transduction from JW5344 \\
\hline AS748 & 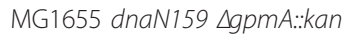 & This study, by P1 transduction from JW0738 \\
\hline AS700 & MG1655 dnaN159 zid501::Tn10 & $\begin{array}{l}\text { This study, by P1 transduction from } \\
\text { NR13339 }\end{array}$ \\
\hline AS717 & MG1655 dnaE486 zae502::Tn10 & This study, by P1 transduction from NR7651 \\
\hline AS765 & MG1655 dnaB8(ts) cmR & This study, by P1 transduction from JJC809 \\
\hline
\end{tabular}

We have tested whether mutations in the CCM genes can suppress temperature sensitivity of bacteria caused by mutations in the replication genes. In this test, bacteria were plated at sublethal temperatures (i.e. temperatures causing a decrease in the efficiency of plating for several orders of magnitude, but still allowing survival of a small fraction of mutant cells), selected on the basis of temperature-sensitivity profiles determined as shown in Figure 1 (in control experiments, the temperature permissive to all strains, $30^{\circ} \mathrm{C}$, was used). These following sublethal temperatures were chosen for particular replication mutants: $39^{\circ} \mathrm{C}$ for $d n a A 46(t s), 41^{\circ} \mathrm{C}$ for $d n a B 8(\mathrm{ts})$, $35^{\circ} \mathrm{C}$ for $d n a C(\mathrm{ts}), 36.5^{\circ} \mathrm{C}$ for $d n a E 486(\mathrm{ts}), 34^{\circ} \mathrm{C}$ for dnaG(ts) and $37.5^{\circ} \mathrm{C}$ for dnaN159(ts).

We found no specific suppression (i.e. suppression which could be reversed by plasmid-mediated expression of the wild-type CCM gene whose defective allele resulted in temperature-tolerance of otherwise temperature-sensitive replication mutant) of the effects of $d n a C$ (ts) mutation by any tested dysfunction in the CCM genes (Figure 3). However, interestingly, efficiency of plating of dnaA46(ts), dnaB8(ts), dnaE486(ts), dnaG(ts) and dnaN159(ts) mutants could be increased by at least one order of magnitude (often considerably more) at sublethal temperatures in the presence of particular mutations in genes coding for enzymes from CCM (Figure 3). The effects of dnaA46(ts) mutation could be suppressed by dysfunction of pta or ackA, effects of dnaB8(ts) by dysfunction of pgi or pta, effects of $d n a E 486$ (ts) by dysfunction of $t k t B$, effects of $d n a G(\mathrm{ts})$ by dysfunction of $g p m A$, pta or ackA, and effects of dnaN159(ts) by dysfunction of pta or ackA. Most of the suppression phenomena were not complete, i.e. the efficiency of survival of the ts mutants in the sublethal temperature was between 1 and $10 \%$ of that in the permissive temperature, though still it was 10 to 100 times higher than that of the ts mutant without suppressor mutation at the sublethal temperature (Figure 4).

Table 2 Plasmids employed and constructed in this study

\begin{tabular}{|c|c|c|}
\hline Plasmid & Relevant characteristics & Reference \\
\hline pBAD24 & Ori pBR322; bla $+P_{B A D}$ & {$[29]$} \\
\hline pAS101 & $\begin{array}{l}\text { pBAD24 bearing the ackA gene } \\
\text { under of pBAD control }\end{array}$ & $\begin{array}{l}\text { This study, by cloning of a PCR amplified fragment of E. coli MG1655 chromosome, obtained with } \\
\text { primers ackaF and ackaR (Table 3), into the Smal side of pBAD24 }\end{array}$ \\
\hline pAS102 & $\begin{array}{l}\text { pBAD24 bearing the pgi gene under } \\
\text { of pBAD control }\end{array}$ & $\begin{array}{l}\text { This study, by cloning of a PCR amplified fragment of E. coli MG1655 chromosome fragment } \\
\text { obtained with primers pgiF and pgiR (Table 3), into the Smal side of pBAD24 }\end{array}$ \\
\hline pAS103 & $\begin{array}{l}\text { pBAD } 24 \text { bearing the } f b a B \text { gene } \\
\text { under of pBAD control }\end{array}$ & $\begin{array}{l}\text { This study, by cloning of a PCR amplified fragment of E. coli MG1655 chromosome fragment } \\
\text { obtained with primers fbabF and fbabR (Table 3), into the Kpnl side of pBAD24 }\end{array}$ \\
\hline pAS104 & $\begin{array}{l}\text { pBAD24 bearing the tktB gene } \\
\text { under of pBAD control }\end{array}$ & $\begin{array}{l}\text { This study, by cloning of a PCR amplified fragment of E. coli MG1655 chromosome fragment } \\
\text { obtained with primers tktbF and tktbR (Table 3), into the Kpnl side of pBAD24 }\end{array}$ \\
\hline pAS105 & $\begin{array}{l}\text { pBAD24 bearing the pta gene under } \\
\text { of pBAD control }\end{array}$ & $\begin{array}{l}\text { This study, by cloning of a PCR amplified fragment of E. coli MG1655 chromosome fragment } \\
\text { obtained with primers ptaF and ptaR (Table 3), into the Kpnl side of pBAD24 }\end{array}$ \\
\hline pAS106 & $\begin{array}{l}\text { pBAD24 bearing the gpm gene } \\
\text { under of pBAD control }\end{array}$ & $\begin{array}{l}\text { This study, by cloning of a PCR amplified fragment of E. coli MG1655 chromosome fragment } \\
\text { obtained with primers gpmaF and gpmaR (Table 3), into the Kpnl side of pBAD24 }\end{array}$ \\
\hline pAS107 & $\begin{array}{l}\text { pBAD24 bearing the } a c e B \text { gene } \\
\text { under of pBAD control }\end{array}$ & $\begin{array}{l}\text { This study by cloning of a PCR amplified fragment of E. coli MG1655 chromosome fragment } \\
\text { obtained with primers acebF and acebR (Table 3), into the Kpnl side of pBAD24 }\end{array}$ \\
\hline
\end{tabular}


Table 3 Oligonucleotides used for cloning

\begin{tabular}{|c|c|c|c|}
\hline Primer name & Primer sequence $\left(5^{\prime}>3^{\prime}\right)$ & $\mathrm{Tm}^{\circ} \mathrm{C}$ & Restriction enzyme site \\
\hline ackaF & GGCCCGGGATGTCGAGTAAGTTAG & 58.0 & Smal \\
\hline ackaR & TGGCAAGCTIACATTCAGGCAGTCAGGCGGCTCG & 60.0 & HindllII \\
\hline gpmaF & CCGGGTACCATGGCTGTAACTAAGCTGGTTCTG & 66.9 & Kpnl \\
\hline gpmaR & CGCGGTCGACTTACTTCGCTTTACCCTGG & 65.7 & Sall \\
\hline fbabF & TCCGGTACCATGACAGATATTGCGCAGTTGCTTG & 65.6 & Kpnl \\
\hline fbabR & GGCCGTCGACTCAGGCGATAGTAATTTTGC & 64.4 & Sall \\
\hline pgiF & GCCCGGGATGAAAAACATCAATCCAACGCAGACC & 66.8 & Smal \\
\hline pgiR & CGGAAGCTITGATTAACCGCGCCACGCTTTATAG & 65.6 & HindIII \\
\hline ptaF & CGGAGGAGGTACCATGTCCCGTATTATTATG & 63.0 & Kpnl \\
\hline ptaR & GACGAAGCTTAGATTACTGCTGCTGTGCAGAC & 64.4 & HindIII \\
\hline tktbF & CGGAGGGTACCATGTCCCGAAAAGACCTTG & 54.0 & Kpnl \\
\hline tktbR & GCGCAAGCTITCAGGCACCTITCACTCCC & 57.0 & HindllI \\
\hline acebF & GAGCGGTACCATGACTGAACAGGCAACAACAAC & 58.0 & Kpnl \\
\hline acebR & TGTGTCGACTTACGCTAACAGGCGGTAGCCTGG & 58.0 & Sall \\
\hline
\end{tabular}

Sequences of particular oligonucleotides recognized by restriction enzymes listed in corresponding row are underlined.

This correlates with the previous findings on the B. subtilis model [13]. Interestingly, the only exceptions were dnaA46 suppressors, restoring $100 \%$ growth relative to that under permissive conditions. It is worth noting that dnaA mutants of B. subtilis were not tested in the previous work, mentioned above [13].

To test whether suppressions depicted in Figure 3 were specific, plasmids bearing wild-type copies of disrupted metabolic genes (Table 2) have been introduced into cells of the double mutants. The wild-type alleles were under control of the pBAD promoter, which could be stimulated by addition of L-arabinose into growth medium. We found that for dnaA46(ts), dnaB8 (ts), dnaE486(ts), dnaG(ts) and dnaN159(ts) hosts, expression of appropriate wild-type allele of CCM gene reversed effects of temperature sensitivity suppression by the corresponding mutant allele (Figure 4). Therefore, we conclude that the suppression effects depicted in Figure 3 are specific for certain mutations.

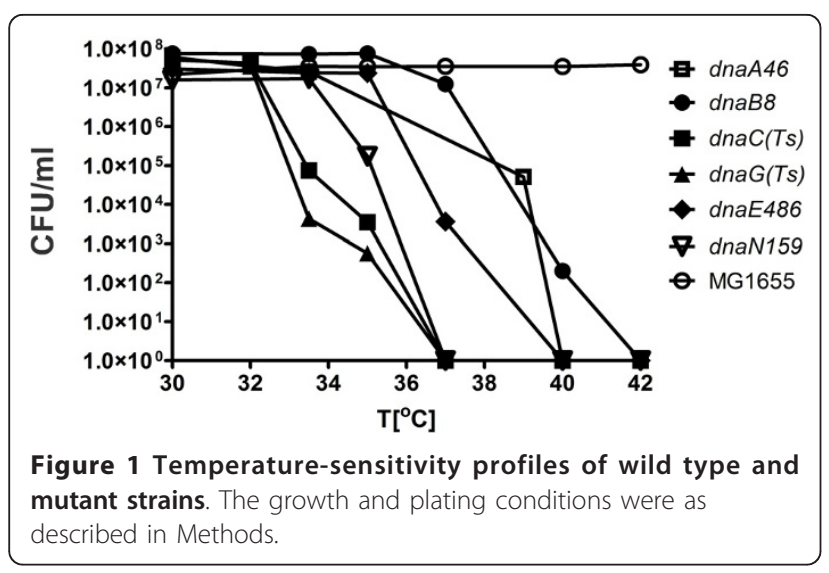

We asked whether the suppression of temperature sensitivity of mutants in the replication genes by dysfunction of particular genes coding for CCM enzymes could be caused by decreased growth rates of double mutants. This question was substantiated by the fact that DNA replication regulation is known to be dependent on bacterial growth rate [2]. However, we found that although in most cases (excluding the $d n a A 46$ mutants) at $30^{\circ} \mathrm{C}$ the growth rates of the double mutants revealing suppression of the temperature sensitivity were lower than in wild-type bacteria, a similar or lower decrease in the growth rate was observed also in double mutants which did not suppress the temperature sensitivity (Figure 5 ). Therefore, we conclude that the observed suppression effects could not be caused simply by a decrease in bacterial growth rate.

We have also tested whether the suppression can be caused by growth of the replication mutants in media containing various carbon sources, which also allow for different growth rates. Therefore, we have plated dnaA46(ts), dnaB8(ts), dnaC(ts), dnaE486(ts), dnaG(ts) and $d n a N 159$ (ts) mutants on plates containing a minimal medium supplemented with various carbon sources: glucose, glycerol, maleic acid or sodium acetate. However, in these experiments, we did not observe any improvement in viability of these mutants at the sublethal temperatures (data not shown). These results corroborate the results of experiments with growth rate measurement, and support our conclusion that the suppression of temperature sensitivity of the replication mutants cannot be explained by lower growth rates of bacteria.

\section{Discussion}

The approach to understand cellular processes as a network of complex relations becomes more appreciated 
Table 4 Enzymes of CCM, whose genes were tested in this study

\begin{tabular}{llll}
\hline EC number & Name & Gene & Pathway \\
\hline EC 1.2.1.9 & Glyceraldehyde-3-phosphate dehydrogenase & gapC & glycolysis/gluconeogenesis \\
EC 2.7.1.40 & Pyruvate kinase & tpiA & \\
EC 5.3.1.1 & Triose-phosphate isomerase & pgi & \\
EC 5.3.1.9 & Glucose-6-phosphate isomerase & fbaB & \\
EC 4.1.2.13 & Fructose-bisphosphate aldolase & gpmA & \\
EC 5.4.2.1 & Phosphoglyceromutase & pckA & \\
EC 4.1.1.49 & Phosphoenolpyruvate carboxykinase (ATP) & zwf & pentose phosphate pathway \\
\hline EC 1.1.1.49 & Glucose-6-phosphate 1-dehydrogenase & tktB & \\
EC 2.2.1.1 & Transketolase B & pta & overflow pathway \\
\hline EC 2.3.1.8 & Phosphate acetyltransferase & ackA & \\
EC 2.7.2.1 & Acetate kinase & aceF & \\
EC 2.3.1.12 & Dihydrolipoyllysine-residue acetyltransferase & aceB & citrate cycle (TCA cycle) \\
\hline EC 2.3.3.9 & Malate synthase & acnB & \\
EC 4.2.1.3 & Aconitate hydratase & $i c d A$ & $i c d C$ \\
EC 1.1.1.42 & Isocitrate dehydrogenase, specific for NADP+ & \\
- & Conserved hypothetical protein (pseudogene) & & \\
\hline
\end{tabular}

only nowadays. Two major processes responsible for maintenance and reproduction of the cell (i.e. energy metabolism and DNA replication) were studied mostly independently until recently. A direct link between DNA replication and central carbon metabolism (CCM) has been demonstrated solely for one species of Grampositive bacterium, B. subtilis [13]. This finding was a breakthrough in considering these processes as interrelated. Thus, it was crucial to address the question whether such a phenomenon occurs only in the specific strain or it is more general. Here we present evidence that such a link exists also in E. coli, a model Gramnegative bacterium.

Despite the general similarity, there are important differences between suppression of effects of mutations in replication genes by dysfunction of genes coding for enzymes of CCM in E. coli and B. subtilis. According to previous report [13], in B. subtilis, the temperature-sensitivity suppression was detected for only three genes: dnaE, dnaC (an equivalent of the E. coli dnaB gene, coding for helicase) and $d n a G$. Temperature-sensitive mutants in these genes could grow at elevated temperatures in the presence of additional mutations in $\operatorname{gap} A$, $p g k, p g m$, eno or pykA. These five genes code for enzymes acting at the late stages of glycolysis and gluconeogenesis. In $E$. coli, we were able to observe suppression of effects of temperature-sensitive mutations not only in dnaE, dnaB and $d n a G$ genes (like in B. subtilis), but also in $\operatorname{dnaN}$ and - perhaps the most surprisingly in $d n a A$. Moreover, growth at sublethal temperatures of these mutants was observed under conditions of a lack of enzymes involved not only in glycolysis and gluconeogenesis (pgi and $g p m A$ ), but also in other regimens of CCM, namely the pentose phosphate pathway $(t k t B$ gene) and the overflow pathway (pta and ackA genes). This suggests that in $E$. coli the link between DNA replication and CCM may be broader than in B. subtilis. Alternatively, the observed differences might result from a partial exploration of a complex system (only some replication and metabolic genes were tested due to technical reasons, namely unavailability of viable mutants).

For B. subtilis, the target of the regulation by metabolic-related signals was shown to be mostly the elongation of the DNA replication process, though some suppressed replication mutations affected also replication initiation [13]. In E. coli, the evidence presented here shows the link between CCM and replication elongation (represented by enzymes involved in the replication complex), and initiation. One of indispensable regulators of the latter process in E. coli is DnaA protein [15,4]. Thus, the finding of the suppression of dnaA46(ts) conditionally-lethal phenotype by mutants in genes involved in CCM suggests the presence of as yet unidentified correlation. Moreover, the observed suppression was complete (100\% survival at sublethal temperature relative to survival at permissive temperature), contrary to those noted for other mutants in replication genes. Both suppressors of the dnaA46(ts) phenotype map in the overflow pathway of CCM. This and the presence of the suppressors in genes of enzymes from other pathways beside glycolysis in $E$. coli could be explained by (i) partial exploration of the coupling system, (ii) the differences in the replication complexes in E. coli and B. subtilis, and/or (iii) different lifestyles and nutrient requirements of these bacterial species. E. coli, during its life cycle, may be exposed to the abrupt changes in the nutrient availability (the "feastfamine" scenario), which requires a more strict regulation, linking energy turnover and DNA replication, thus, 


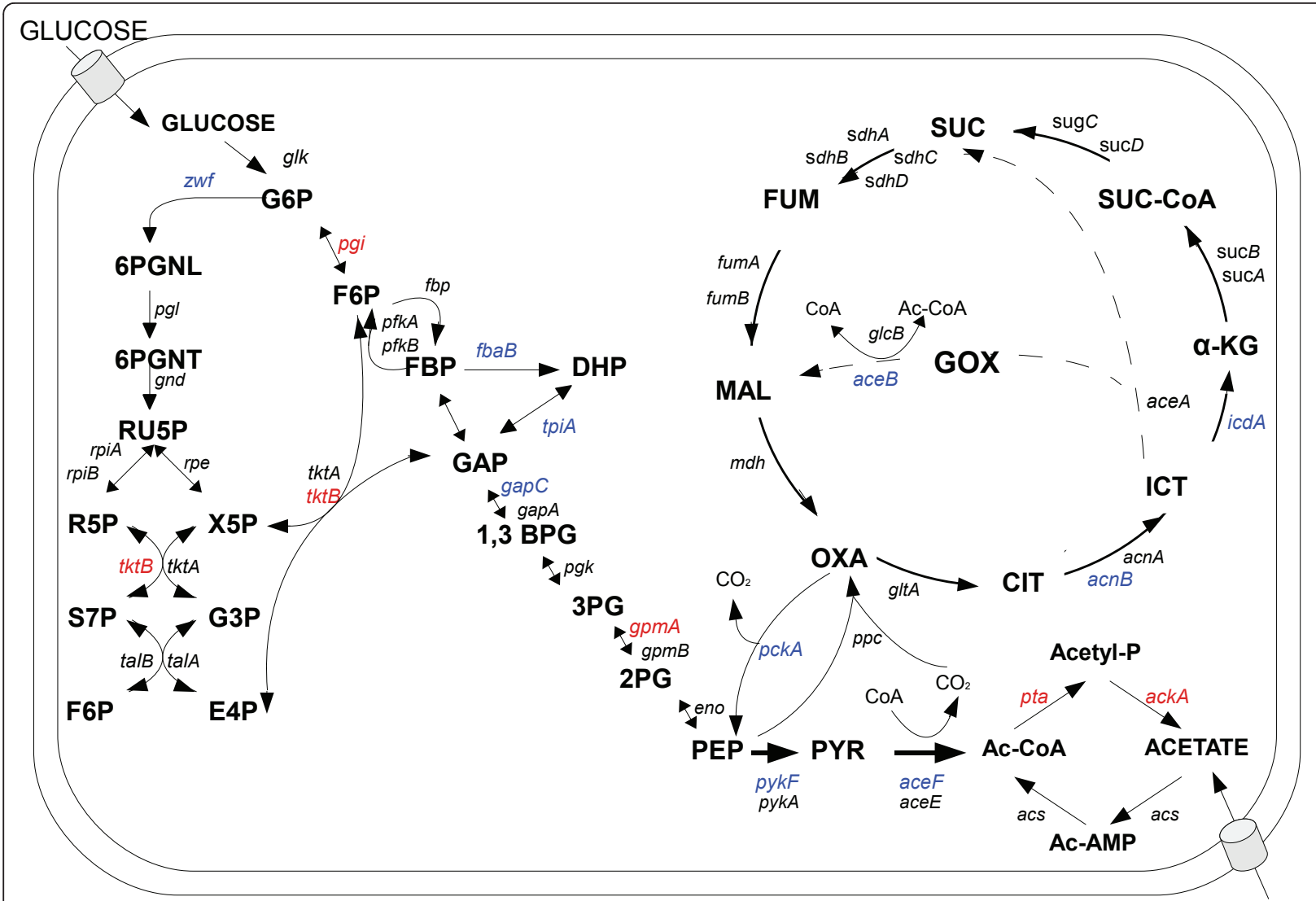

ACETATE

Figure 2 A scheme for CCM including main pathways - glycolysis/gluconeogenesis, penthaphosphate pathway, citrate cycle, overflow pathway. Mutants tested in this work are indicated by following colours: blue - non suppressor mutants, red - suppressors of replication genes mutants. Metabolites abbreviations: 1,3-BGP, 1,3-biphosphoglycerate; 2PG, 2-phophoglycerate; 3PG, 3-phosphoglycerate; 6PGLN, 6phosphoglucono- $\delta$-lactone; 6 PGNT, 6-phophogluconate; GLC, glucose; G6P, glucose-6-phosphate; F6P, fructose-6-phosphate; FUM, fumarate; MAL, malate; OXA, oxaloacetate PBP, fructose-1,6-biphosphate; DHAP, dihydroxyacetone phosphate; GAP, glyceraldehyde 3-phosphate; PEP, phosphoenolpyruvate; PYR, pyruvate; Ru5P, ribulose-5-phosphate; R5P, ribose-5-phosphate; S7P, sedoheptulose-7-phosphate; E4P, erythrose-4phosphate; Ac-CoA, acetyl coenzyme A; Ac-P, acetyl phosphate; Ac-AMP, acetyl-AMP; CIT, citrate; ICT, isocitrate; GOX, glyoxylate; $\alpha-K G, \alpha-$ ketoglutarate; SUC-CoA, succinyl-coenzyme A; SUC, succinate; Xu5P, xylulose-5-phosphate.

\begin{tabular}{|c|c|c|c|c|c|c|c|c|c|c|c|c|c|c|c|}
\hline & \multicolumn{7}{|c|}{ Glycolysis/gluconeogenesis } & \multicolumn{2}{|c|}{ PPP } & \multicolumn{2}{|c|}{ Overflow } & \multicolumn{4}{|c|}{ Krebs cycle } \\
\hline & gapC & pykF & tpiA & pgi & $f b a B$ & gpmA & pck & $z w f$ & $t k t B$ & pta & ackA & aceB & $a c n B$ & icd & $i c d C$ \\
\hline \multicolumn{16}{|l|}{ dnaA } \\
\hline \multicolumn{16}{|l|}{ dnaB } \\
\hline \multicolumn{16}{|l|}{ dnaC } \\
\hline \multicolumn{16}{|l|}{ dnaE } \\
\hline \multicolumn{16}{|l|}{ dnaG } \\
\hline dnaN & & & & & & & & & & & & & & & \\
\hline
\end{tabular}




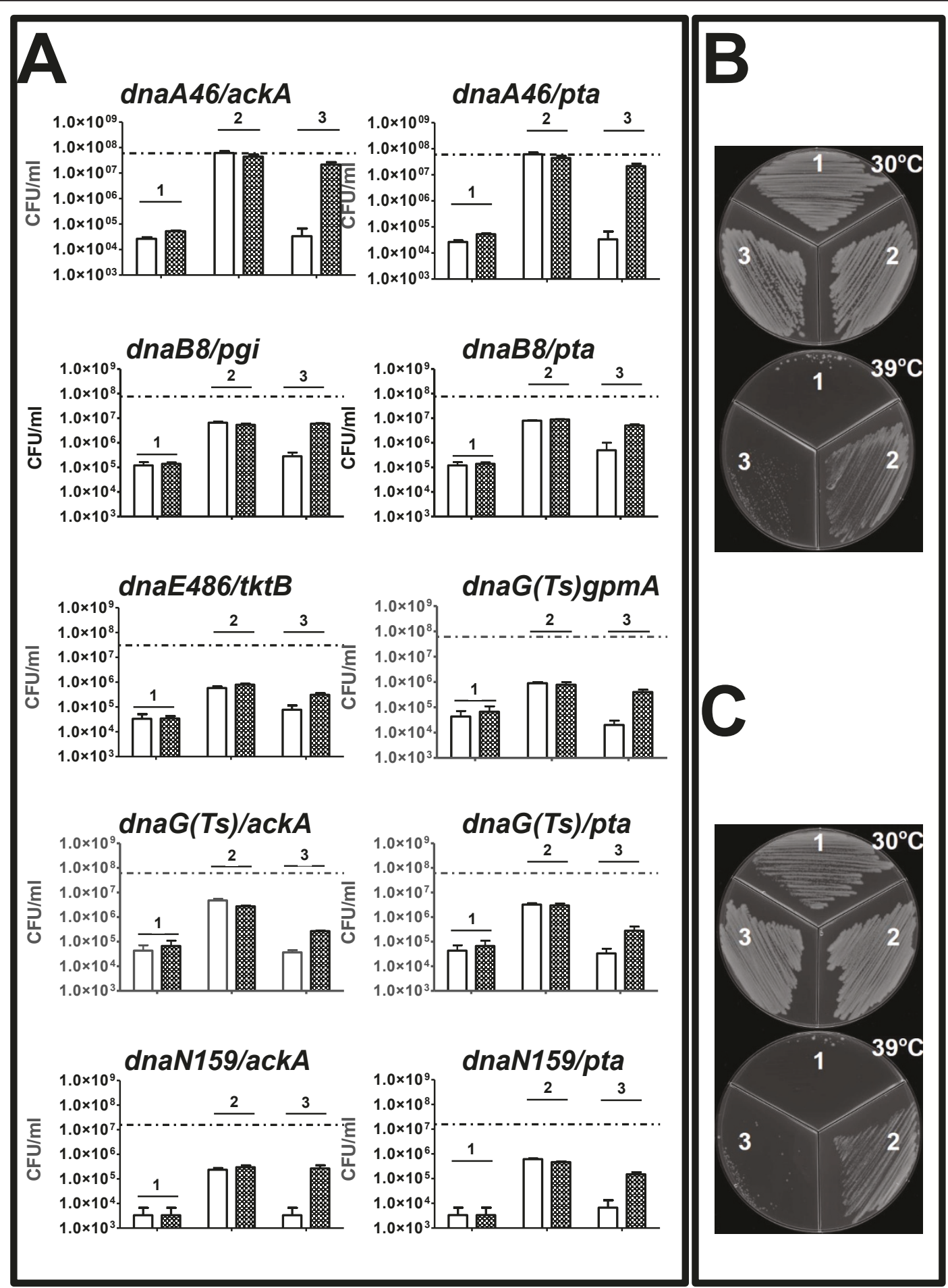

Figure 4 Complementation of suppression phenotypes in double replication/CCM mutants by the overproduction of the metabolic enzymes. The experiments were performed in sublethal temperatures (relevant for each strain). Mutations as indicated above the graphs were employed. Panel A. Bacterial growth measured in CFU. Empty columns - growth in the presence of $0.2 \%$ arabinose, shaded columns - growth in the presence of $0.1 \%$ glucose. Efficiencies of plating $(\mathrm{CFU} / \mathrm{ml})$ of the replication mutants at $30^{\circ} \mathrm{C}$ are indicated by a dashed line at each graph. Panel B and C. The growth of temperature sensitive dnaA46-derivatives in permissive and sublethal temperature. B - dnaA464pta, C dnaA46 $\triangle a c k$ A. Panels A, B and C. 1 - temperature-sensitive replication mutants, 2 - double mutants in replication and CCM genes, 3 - double mutants in replication and CCM genes complemented with the relevant metabolic gene under the control of arabinose-inducible pBAD promoter 


\begin{tabular}{|c|c|c|c|c|c|c|c|c|c|}
\hline \multirow{2}{*}{$\begin{array}{l}\text { Replication } \\
\text { mutants }\end{array}$} & \multicolumn{9}{|c|}{ Mutation in metabolic gene } \\
\hline & None & tpiA & pgi & $f b a B$ & gpmA & $t k t B$ & pta & $\operatorname{ack} A$ & $a c e B$ \\
\hline dnaA46 & $52 \pm 7.6$ & - & $35 \pm 0.0$ & - & - & - & $38 \pm 7.6$ & $37 \pm 3.8$ & - \\
\hline dnaB8 & $56 \pm 5.8$ & $46 \pm 6.4$ & $115 \pm 0.0$ & - & - & - & $50 \pm 0.0$ & - & - \\
\hline$d n a C(T s)$ & $48 \pm 0,7$ & - & $42 \pm 2.1$ & $75 \pm 0.0$ & $45 \pm 8.3$ & $65 \pm 7.0$ & - & - & - \\
\hline dnaE486 & $37 \pm 2.8$ & - & - & $24 \pm 5.0$ & - & $42 \pm 4.2$ & - & - & - \\
\hline$d n a G(T s)$ & $43 \pm 3.4$ & $55 \pm 7.6$ & - & - & $44 \pm 12.8$ & - & $53 \pm 6.6$ & $52 \pm 7.1$ & $41 \pm 5.3$ \\
\hline$d n a N 159$ & $43 \pm 2.5$ & - & - & $73 \pm 3.5$ & - & - & $46 \pm 8.5$ & $50 \pm 7.0$ & - \\
\hline \multicolumn{10}{|c|}{$\begin{array}{l}\text { Figure } 5 \text { Generation times of double mutants in replication and CCM genes. Bacteria were grown at } 30^{\circ} \mathrm{C} \text { in } \mathrm{LB} \text { and doubling time (values } \\
\text { presented in the boxes } \pm \mathrm{SD} \text { ) was assessed in the exponential growth phase. The doubling time for the wild-type strain (MG1655) was } 48 \pm 0.7 \\
\text { min. The colors represent genotypes in which suppressions were observed at sublethal temperatures (red - full suppression, yellow - incomplete } \\
\text { suppression). Dash - the generation time was not determined. }\end{array}$} \\
\hline
\end{tabular}

it may profit from more metabolic sensors. Similarly to $B$. subtilis, the suppression observed in E. coli was not caused by a decrease in the growth rate. Moreover, the increase in the doubling time of replication mutants (by growth on the minimal media containing various carbon sources, including very poor ones, like maleic acid or acetate) did not improve their viability at sublethal temperatures.

The proposed mechanism of the regulation of DNA replication by CCM in $B$. subtilis involves a putative metabolic linker which can cause conformational changes in replication proteins to modulate replisome properties [13]. This hypothesis may be supported by the role of acetyl phosphate which can accumulate in the overflow pathway mutants. Acetyl phosphate has been proposed to function as a global signal that fits into various two-compound systems $[16,17]$. This may require the second, as yet unknown, protein modulating replication proteins, or the mechanism can rely on autophosphorylation. The role of acetyl phosphate in protein folding and stability has been proposed as well [18]. In this light it is interesting that AckA and Pta reduce the production of double-stranded breaks in DNA [19]. Moreover, DiaA, a DnaA-binding protein, contains a SIS motif that might bind phosphosugars [20]. These facts may provide a start point to further works on understanding the link between CCM and DNA replication.

It is worth noting that since we have used deletioninsertion mutants in genes coding for CCM enzymes, the suppressions of the temperature-sensitivity phenotypes of the replication mutants cannot be explained by direct protein-protein interactions. Indeed, numerous and large-scale interactions between replication proteins and CCM enzymes seemed unlikely, which led us to use a set of deletion mutants in tested genes. On the other hand, the use of such mutants ensured that particular enzymatic functions were absent in mutant cells, which excluded potential problems with putative partial inactivation of CCM enzymes caused by point mutations.

One should also take into consideration a possibility that changes in chemical composition of the cells caused by a lack of particular CCM enzymes might alleviate temperature sensitivity of mutants in genes coding for replication proteins. In fact, we cannot exclude that increased concentrations of some substances that accumulate due to metabolic blocks at certain steps of CCM might stabilize the temperature-sensitive replication proteins and allow them to function at higher temperatures. If so, CCM could have no effects on wild-type replication proteins and the DNA replication process in wildtype cells. However, to accept such a hypothesis it would be necessary to assume that there are at least several compounds (metabolites) able to interact specifically with several different temperature-sensitive variants of the replication proteins, resulting in their stabilization at elevated temperatures. Although still possible, such a scenario seems unlikely, therefore, we prefer the hypothesis that there is a link between CCM and DNA replication in bacterial cells.

\section{Conclusions}

We show the genetic correlation between central carbon metabolism and DNA replication in the model Gramnegative bacterium, E. coli. Therefore, one might suggest 
that the existence of such a link is a general phenomenon rather than an event occurring very specifically in a small group of organisms. This link exists at the steps of initiation and elongation of DNA replication, indicating the important global correlation between metabolic status of the cell and the events leading to cell reproduction.

\section{List of abbreviations}

CFU: colony forming unit; CCM: central carbon metabolism; PPP: pentose phosphate pathway; ts: temperature-sensitivity.

\section{Acknowledgements and Funding}

We are grateful to Dr. Benedicte Michel and Dr. Iwona Fijałkowska for replication mutant strains. The mutants in the CCM genes were obtained from the Keio collection (25, National BioResource Project (NIG, Japan): E. coli). This work was supported by Ministry of Science and Higher Education (Poland) (project grant no. N N301 467234 to GW).

\section{Author details}

${ }^{1}$ Department of Molecular Biology, University of Gdańsk, Kładki 24, 80-822 Gdańsk, Poland. ${ }^{2}$ MEGA Laboratory, Institute of System and Synthetic Biology, Génopole Campus I, 5 rue Henri Desbruères, 91000 Evry, France.

\section{Authors' contributions}

MM and DN performed all experiments. $\amalg$ was the initiator of the project and contributed to experimental design and data analysis. ASP supervised experiments and participated in preparation of the manuscript. GW was a project leader, supervised the work and drafted the manuscript. All authors read and approved the final manuscript.

\section{Competing interests}

The authors declare that they have no competing interests.

Received: 11 February 2011 Accepted: 31 March 2011

Published: 31 March 2011

\section{References}

1. Gottschalk G: Bacterial Metabolism. Springer, Berlin-Heidelberg; 21986.

2. Kornberg A, Baker TA: DNA Replication. University Science Books, Sausalito, $C A_{i} 21992$.

3. Neidhardt FC: Escherichia coli and Salmonella Cellular and Molecular Biology. ASM Press. Washington, D.C.; 2 1996:2:189-206.

4. Zakrzewska-Czerwińska J, Jakimowicz D, Zawilak-Pawlik A, Messer W Regulation of the initiation of chromosomal replication in bacteria. FEMS Microbiol Rev 2007, 31:378-387.

5. Zyskind JW, Smith DW: DNA replication, the bacterial cell cycle, and cell growth. Cell 1992, 69:5-8.

6. Michelsen O, Teixeira de Mattos MJ, Jensen PR, Hansen FG: Precise determinations of $C$ and $D$ periods by flow cytometry in Escherichia coli K-12 and B/r. Microbiology 2003, 149:1001-1010.

7. Hughes $P$, Landoulsi A, Kohiyama M: A novel role for CAMP in the control of the activity of the $E$. coli chromosome replication initiator protein, DnaA. Cell 1988, 55:343-350.

8. Landoulsi A, Kohiyama M: Initiation of DNA replication in $\triangle$ cya mutants of Escherichia coli K12. Biochimie 1999, 81:827-834.

9. Levine A, Vannier F, Dehbi M, Henckes G, Seror SJ: The stringent response blocks DNA replication outside the ori region in Bacillus subtilis and at the origin in Escherichia coli. J Mol Biol 1991, 219:605-613.

10. Schreiber G, Ron EZ, Glaser G: ppGpp-mediated regulation of DNA replication and cell division in Escherichia coli. Curr Microbiol 1995, 30:27-32.

11. Herman A, Wegrzyn G: Effect of increased ppGpp concentration on DNA replication of different replicons in Escherichia coli. J Basic Microbiol 1995, 35:33-39.

12. Wegrzyn G, Wegrzyn A: Stress responses and replication of plasmids in bacterial cells. Microb Cell Fact 2002, 1:2.
13. Jannière L, Canceill D, Suski C, Kanga S, Dalmais B, Lestini R, Monnier AF, Chapuis J, Bolotin A, Titok M, Le Chatelier E, Ehrlich SD: Genetic evidence for a link between glycolysis and DNA replication. PLOS ONE 2007, 2:e447.

14. Sambrook J, Russell DW: Molecular Cloning: A Laboratory Manual. Cold Spring Harbor Laboratory Press. Cold Spring Harbor, New York; 32001.

15. Messer W: The bacterial replication initiator DnaA. DnaA and oriC, the bacterial mode to initiate DNA replication. FEMS Microbiol Rev 2002, 26:355-374.

16. Klein AH, Shulla A, Reimann SA, Keating DH, Wolfe AJ: The intracellular concentration of acetyl phosphate in Escherichia coli is sufficient for direct phosphorylation of two-component response regulators. $J$ Bacteriol 2007, 189:5574-5581

17. McCleary WR, Stock JB: Acetyl phosphate and the activation of twocomponent response regulators. J Biol Chem 1994, 269:31567-31572.

18. Mizrahi I, Biran D, Ron EZ: Involvement of the Pta-AckA pathway in protein folding and aggregation. Res Microbiol 2009, 160:80-84.

19. Shi IY, Stansbury J, Kuzminov A: A defect in the acetyl coenzyme A acetate pathway poisons recombinational repair-deficient mutants of Escherichia coli. J Bacteriol 2005, 187:1266-1275.

20. Ishida T, Akimitsu N, Kashioka T, Hatano M, Kubota T, Ogata Y, Sekimizu K, Katayama T: DiaA, a novel DnaA-binding protein, ensures the timely initiation of Escherichia coli chromosome replication. J Biol Chem 2004, 279:45546-45555.

21. Carl PL: Escherichia coli mutants with temperature-sensitive synthesis of DNA. Mol Gen Genet 1970, 109:107-122.

22. Dabbs ER: The gene for ribosomal protein $\mathrm{S} 21, r p s U$, maps close to dnaG at 66.5 min on the Escherichia coli chromosomal linkage map. J Bacteriol 1980, 144:603-607.

23. Jensen KF: The Escherichia coli K-12 "wild types" W3110 and MG1655 have an rph frameshift mutation that leads to pyrimidine starvation due to low pyrE expression levels. J Bacteriol 1993, 175:3401-3407.

24. Fayet O, Louarn JM, Georgopoulos C: Suppression of the Escherichia coli dnaA46 mutation by amplification of the groES and groEL genes. $\mathrm{Mol}$ Gen Genet 1986, 202:435-445.

25. Seth GN, Grant T, Jesseet J, Bloomt FR, Hanahan D: Differential plasmid rescue from transgenic mouse DNAs into Escherichia coli methylationrestriction mutants (bacterial restriction/DNA methylation/cloning mammalian DNA/heterogeneous transgene expression/insulin gene regulation). Proc Natl Acad Sci USA 1990, 87:4645-4649.

26. Datsenko KA, Wanner BL: One-step inactivation of chromosomal genes in Escherichia coli K-12 using PCR products. Proc Natl Acad Sci USA 2000, 97:6640-6645.

27. Baba T, Ara T, Hasegawa M, Takai Y, Okumura Y, Baba M, Datsenko KA Tomita M, Wanner BL, Mori H: Construction of Escherichia coli K-12 inframe, single-gene knockout mutants: the Keio collection. Mol Syst Biol 2006, 2:2006-0008.

28. Makiela-Dzbenska $K$, Jaszczur M, Banach-Orlowska M, Jonczyk $P$, Schaaper RM, Fijalkowska IJ: Role of Escherichia coli DNA Polymerase I in chromosomal DNA replication fidelity. Mol Microbiol 2009, 74:1114-1127.

29. Guzman LM, Belin D, Carson M, Beckwith J: Tight regulation, modulation and high-level expression by vectors containing the arabinose PBAD promoter. J. Bacteriol 1995, 177:4121-4130.

doi:10.1186/1475-2859-10-19

Cite this article as: Maciag et al:: Genetic response to metabolic fluctuations: correlation between central carbon metabolism and DNA replication in Escherichia coli. Microbial Cell Factories 2011 10:19. 